\title{
Molecular and Serological Intraocular Fluid Analysis of Coxiella burnetii-seropositive Patients with Concurrent Idiopathic Uveitis
}

L. E. Hermans, J. J. Oosterheert, L. M. Kampschreur, J. Ossewaarde-van Norel, J. Dekkers, A. Rothova \& J. D. F. de Groot-Mijnes

To cite this article: L. E. Hermans, J. J. Oosterheert, L. M. Kampschreur, J. Ossewaarde-van Norel, J. Dekkers, A. Rothova \& J. D. F. de Groot-Mijnes (2016) Molecular and Serological Intraocular Fluid Analysis of Coxiella burnetii-seropositive Patients with Concurrent Idiopathic Uveitis, Ocular Immunology and Inflammation, 24:1, 77-80, DOI: 10.3109/09273948.2014.925123

To link to this article: http://dx.doi.org/10.3109/09273948.2014.925123

Published online: 19 Jun 2014.

Submit your article to this journal

Џلll Article views: 104

Q View related articles $\square$

View Crossmark data ¿ 


\title{
Molecular and Serological Intraocular Fluid Analysis of Coxiella burnetii-seropositive Patients with Concurrent Idiopathic Uveitis
}

\author{
L. E. Hermans, $\mathrm{MS}^{1,2}$, J. J. Oosterheert, $\mathrm{MD}, \mathrm{PhD}^{3}$, L. M. Kampschreur, $\mathrm{MD} \mathrm{PhD}^{3}$, \\ J. Ossewaarde-van Norel, $\mathrm{MD}^{2}$, J. Dekkers, $\mathrm{MS}^{1,2}$, A. Rothova, $\mathrm{MD}, \mathrm{PhD}^{2,4}$, \\ J. D. F. de Groot-Mijnes, $\mathrm{MD}, \mathrm{PhD}^{1,2}$ \\ ${ }^{1}$ Department of Virology, ${ }^{2}$ Department of Ophthalmology, ${ }^{3}$ Department of Internal Medicine and Infectious \\ Diseases, ${ }^{4}$ Department of Ophthalmology, Erasmus Medical Center, Rotterdam, The Netherlands
}

\begin{abstract}
Purpose: Previous studies have suggested a link between $\mathrm{Q}$ fever and uveitis. We determined whether Coxiella burnetii causes intraocular infection in C. burnetii-seropositive patients with idiopathic uveitis.

Methods: From a retrospective observational case series, paired aqueous humor and serum samples from $10 \mathrm{C}$. burnetii-seropositive patients with idiopathic uveitis were examined for intraocular antibody production by using the Goldmann-Witmer coefficient and by polymerase chain reaction (PCR).

Results: Although intraocular IgG against C. burnetii was detected, no intraocular antibody production was observed (low Goldmann Wittmer coefficients). All PCR results were negative.

Conclusions: Uveitis due to an intraocular infection with C. burnetii is unlikely.
\end{abstract}

Keywords: Aqueous humor analysis, Coxiella burnetii, infection, uveitis

Uveitis may be caused by intraocular infection or systemic (autoimmune) disease, ${ }^{1,2}$ but remains idiopathic in approximately a quarter of cases. Intraocular fluid analysis by both polymerase chain reaction (PCR) and detection of intraocular antibody production is of great value to distinguish infectious from noninfectious uveitis. ${ }^{3}$ Moreover, it has led to the discovery of new and putative infectious causes of uveitis. $^{4,5}$

Several cases have been described suggesting that Coxiella burnetii, the causative agent of $\mathrm{Q}$ fever, may be involved in the development of uveitis. $6,7,8,9,10$ In addition, one study reported the identification of 9 C. burnetii seropositive cases in a large cohort of 1520 idiopathic uveitis patients. While C. burnetiispecific PCR analysis of anterior chamber fluid of patients with a positive $Q$ fever serology was negative, the authors hypothesized that C. burnetii caused uveitis in these cases in an antibody-mediated manner. ${ }^{11,12}$ Determination of intraocular antibody production against $C$. burnetii, which could confirm this hypothesis, has not been reported yet. From mid 2007 till 2010 a Q fever epidemic occurred in the Netherlands which was, with over 4000 reported acute $\mathrm{Q}$ fever cases (which is an underestimation of the real magnitude of the outbreak), unmatched worldwide in size and duration. ${ }^{13}$ This event allowed us to investigate the association between Coxiella burnetii and uveitis more closely. Using PCR and Goldmann-Witmer coefficient (GWC) analysis to detect intraocular antibody production, we set out to provide evidence for or against the hypothesis that Coxiella burnetii is causally related to uveitis. 


\section{METHODS}

\section{Patients and Samples}

Patients were selected from a database of paired aqueous humor and serum samples that were collected for diagnostic purposes between 1 January 2008 and 31 December 2010 at the University Medical Center Utrecht, a nationwide reference center for the diagnosis of infectious uveitis. The intraocular fluid samples had been stored at $-80^{\circ} \mathrm{C}$ within $5 \mathrm{~h}$ of collection prior to processing for laboratory analysis. All samples had been analyzed by PCR and GWC analysis for at least herpes simplex virus 1 and 2, varicella zoster virus, and Treponema pallidum, and in case of posterior uveitis also Toxoplasma gondii. We hypothesized that putative cases of C. burnetiiinduced uveitis would be far more likely to occur in those patients where no other infectious cause for the uveitis has been found. Patients under the age of 18 years or with a laboratory-confirmed infectious uveitis caused by cytomegalovirus, herpes simplex virus types 1 and 2, varicella zoster virus, rubella virus, or Toxoplasma gondii were excluded. HLA-B27positive patients and patients with sarcoidosis were not excluded, as in these cases an infectious trigger has been suggested.

To identify possible cases of uveitis and simultaneous $C$. burnetii infection, each patient's municipality of residence and year of first presentation with uveitis was compared to epidemiological data on the incidence of $Q$ fever. Serological screening for $Q$ fever was performed in all those cases where patients presented with uveitis while their municipality of residence was affected by the epidemic. Intraocular fluids from all $C$. burnetii-seropositive patients with idiopathic uveitis were analyzed by PCR and intraocular antibody production analysis. This study was performed according to the tenets of the Declaration of Helsinki and in agreement with the regulations of the institutional review board of the Utrecht University Medical Center (UMCU). All patients consented to the use of their materials for research purposes.

\section{Antibody Detection}

Patients were screened for C. burnetii-specific IgG in serum using the Panbio Coxiella burnetii (Q fever) IgG ELISA (Panbio, Brisbane, Australia) according to the instructions of the manufacturer. Intraocular antibody production was determined by using the GWC analysis essentially as described previously. ${ }^{3}$ C. burnetii IgG titers were determined by analyzing 4 serial fourfold dilutions starting at 1:101 for serum and 4 serial twofold dilutions starting at 1:50.5 for intraocular fluid using the Panbio Coxiella burnetii $(\mathrm{Q}$ fever) IgG ELISA. IgG titers were determined by calculating the dilution at which the extrapolated linear part of the ELISA curves crossed the cutoff value. Total IgG concentrations were determined by an in-house assay as described previously. ${ }^{3}$ The GWC was calculated as follows ([specific IgG eye/total IgG eye]:[specific IgG serum/total IgG serum]). Values exceeding 3 are considered indicative of intraocular antibody production. ${ }^{3}$

\section{PCR Analysis}

Taqman PCR analysis was performed as described previously. ${ }^{3}$ Bacterial DNA was extracted from $12.5 \mu \mathrm{L}$ of ocular fluid using the MagNa Pure LC Total Nucleic Acid isolation kit - large volume (Roche, Almere, The Netherlands). To monitor the quality of the extraction and the subsequent amplification procedure a standard dose of phocine herpesvirus type 1 (PhHV-1) was added to each sample as an internal control prior to extraction. ${ }^{14}$ The DNA was collected in a volume of $100 \mu \mathrm{L}$, and per PCR reaction $10 \mu \mathrm{L}$ was added. The primers and probe for PhHV-1 DNA amplification were described previously. ${ }^{3} \mathrm{C}$. burnetii DNA was amplified using forward primer (5'AAAACGGATAAAAAGAGTCTGTGGTT- $3^{\prime}$ ), reverse primer (5'-CCACACAAGCGCGATTCAT-3') and probe (5'-FAM-AAAGCACTCATTGAGCGCCGCGTAMRA-3') as described in ${ }^{15}$ Taqman PCR was performed on an ABI Prism 7900 HT sequence detection system (Applied Biosystems, Foster City, CA, USA). The analytical sensitivity of the assay was determined to lie between 10 and 30 copies per reaction. The analytical specificity is $100 \%$. All samples were analyzed in duplicate. None of the samples were inhibited (Tilburg 2010).

\section{RESULTS}

A total of 773 adult patients suffering from presumed noninfectious uveitis were included, 245 of which had an elevated risk of Coxiella infection, as was determined by linking municipality to the epidemiological data of the Dutch Q fever epidemic. Of these 245 patients, 85 had a uveitis of undefined etiology, 3 of which had been found $C$. burnetii seropositive previously. Of the remaining 82 patients, sufficient serum for $C$. burnetii antibody screening was available in 72 cases. Of these, 8 were seropositive, yielding $11 \mathrm{C}$. burnetii-seropositive patients with idiopathic uveitis.

In 10 cases sufficient ocular fluid was available for GWC determination. Intraocular IgG and thus the GWC were negative in 8 patients. In 2 cases (1 and 9), intraocular C. burnetii-specific IgG was positive, but the GWC did not exceed 3, indicating that 
TABLE 1. Clinical characteristics and laboratory results of Coxiella burnetii-seropositive patients with idiopathic uveitis.

\begin{tabular}{|c|c|c|c|c|c|c|c|c|}
\hline & Gender & Age & $\begin{array}{c}\text { Year of } \\
\text { sampling }\end{array}$ & Clinical diagnosis & Laterality & Ocular IgG titer & GWC & PCR \\
\hline 1 & Female & 23 & 2010 & Acute multifocal placoid pigment epitheliopathy & Unilateral & $1: 387$ & 1.96 & Neg \\
\hline 2 & Male & 80 & 2010 & Idiopathic panuveitis and fever & Bilateral & Neg & Neg & Neg \\
\hline 3 & Female & 39 & 2009 & Idiopathic anterior uveitis & Bilateral & Neg & Neg & Neg \\
\hline 4 & Female & 69 & 2009 & Anterior uveitis and sarcoidosis & Bilateral & Neg & Neg & Neg \\
\hline 5 & Female & 51 & 2009 & Nodular scleritis and aortitis & Unilateral & Neg & Neg & nd \\
\hline 6 & Male & 43 & 2009 & Idiopathic chorioretinitis & Unilateral & Neg & Neg & Neg \\
\hline 7 & Male & 60 & 2009 & Idiopathic panuveitis & Bilateral & Neg & Neg & Neg \\
\hline 8 & Male & 49 & 2009 & Idiopathic panuveitis & Unilateral & Neg & Neg & Neg \\
\hline 9 & Female & 48 & 2010 & Panuveitis and papillitis & - & $1: 51$ & 2.86 & nd \\
\hline 10 & Male & 66 & 2009 & Idiopathic neuroretinitis & Unilateral & Neg & Neg & Neg \\
\hline
\end{tabular}

GWC, Goldmann-Witmer coefficient; PCR, polymerase chain reaction; nd, not determined.

TABLE 2. Clinical characteristics of patients with concurrent uveitis and Coxiella burnetii seropositivity reported in literature.

\begin{tabular}{lllllll}
\hline Article & Gender & Age & Country & Q fever & Uveitis characteristics & Uveitis entities excluded \\
\hline 6 & Female & 33 & France & No & Anterior unilateral & Toxoplasmosis, rheumatism, TB \\
7 & Male & 22 & France & Yes & Posterior bilateral & Unspecified \\
10 & Female & 75 & France & No & Posterior unilateral & Unspecified \\
9 & Male & 44 & Spain & Yes & Choroidal neovascularization & Unspecified \\
Million et al. 2010 & Male & 58 & France & Yes & Anterior unilateral & Syphilis, TB, sarcoidosis \\
& Male & 56 & France & No & Posterior bilateral, optic & Syphilis, Bartonella henselae/quintana, \\
11,12 & & & & & nouritis & Bnspecia \\
& Male & 43 & France & Unknown & Posterior unilateral & Unspecified \\
& Female & 39 & France & Unknown & Anterior unilateral & Unspecified \\
& Female & 69 & France & Unknown & Anterior unilateral & Unspecified \\
& Female & 57 & France & Unknown & Anterior bilateral & Unspecified \\
& Male & 76 & France & Unknown & Anterior bilateral & Unspecified \\
& Female & 86 & France & Unknown & Anterior unilateral & Unspecified \\
Male & 88 & France & Unknown & Posterior unilateral & Unspecified \\
& Female & 76 & France & Unknown & Posterior unilateral & Unspecified \\
& Male & 59 & France & Unknown & Anterior unilateral & \\
\hline
\end{tabular}

Coxiella-specific IgG was not produced locally, but had leaked from the peripheral blood into the aqueous chamber. PCR analysis was possible on the remainders of 7 aqueous humor samples, but positive results were not found. The clinical characteristics and laboratory results of the examined C. burnetii-seropositive patients are listed in Table 1.

\section{DISCUSSION}

The present study is the first to examine intraocular $C$. burnetii infection by analysis of intraocular fluid samples using both GWC and PCR. Neither intraocular antibody production nor C. burnetii DNA was detected in seropositive patients identified from a cohort of patients with idiopathic uveitis living in the 2007-2010 Q fever epidemic regions in the Netherlands, suggesting that C. burnetii was not the causative agent of the uveitis. Obviously, this study is hampered by the relative low number of $C$. burnetiiseropositive uveitis patients analyzed.
Assuming C. burnetii uveitis does exist, these results could be explained by a low intraocular bacterial load, concomitant with undetectable intraocular antibody production. False-negative antibody results could arise if only anti-phase I antibodies are produced intraocularly, whereas the ELISA applied here detects anti-phase II antibodies. However, this is unlikely, as anti-phase II antibodies are present during both the acute and chronic phases of the disease, whereas anti-phase I antibodies are predominant in chronic disease. ${ }^{16}$ Alternatively, C. burnetii uveitis could result from an autoimmune or autoinflammatory response triggered by the bacterial infection, which is not identifiable by GWC or PCR.

On the other hand, $Q$ fever-associated uveitis may not exist at all. Six Coxiella-seropositive patients with uveitis have been described in 5 medical case reports, $6,7,8,9,10$ and an additional 9 cases were recognized in a large survey. ${ }^{11,12}$ These 15 cases, summarized in Table 2, show varying types of uveitis, occurring either during episodes of $Q$ fever or in asymptomatic seropositive patients, as was the case in our patient series. All but one of the previously 
described cases concern patients from France, a country where $Q$ fever is endemic to many rural regions. Also, in the described cases, it is often unclear if other possible causes of uveitis were ruled out. The combined data, therefore, support the hypothesis that coexistence of $\mathrm{Q}$ fever and uveitis is merely a chance finding.

In conclusion, although it cannot be ruled out that C. burnetii is associated with uveitis, the current combined data suggest that intraocular infection with C. burnetii is an unlikely cause of uveitis.

\section{DECLARATION OF INTEREST}

The authors report no conflicts of interest. The authors alone are responsible for the content and writing of the paper.

This study was in part supported by the Dr. F. P. Fischer Foundation, Amersfoort, The Netherlands, and the Foundation for Dutch Ophthalmic Research (SNOO), Rotterdam, The Netherlands. The authors thank José van der Wal for excellent technical assistance.

Author contributions: Involved in study design (LEH, JJO, LMK, JDFG); conduction of study and data collection ( $\mathrm{LEH}, \mathrm{LMK}, \mathrm{JD}, \mathrm{JO}$ ); analysis and interpretation of data (LEH, JD, JDFG); drafting manuscript (LEH); review and approval of manuscript (JJO, LMK, JO, AR, JDFG).

\section{REFERENCES}

1. de Smet AM, Taylor SR, Bodaghi B, et al. Understanding uveitis: the impact of research on visual outcomes. Prog Retin Eye Res. 2011;30:452-470.

2. Shafik SM, Foster C. (2002). Definition, classification, etiology, and epidemiology. In Foster VA, ed. Diagnosis and Treatment of Uveitis. Philadelphia: WB Saunders; 2002:17-26.

3. de Groot-Mijnes JD, Rothova A, van Loon AM, et al. Polymerase chain reaction and Goldmann-Witmer coefficient analysis are complimentary for the diagnosis of infectious uveitis. Am J Ophthalmol. 2006;141:313-318.

4. de Groot-Mijnes JD, de Visser L, Zuurveen S, et al. Identification of new pathogens in the intraocular fluid of patients with uveitis. Am J Ophthalmol. 2010;150:628-636.

5. Quentin CD, Reiber H. Fuchs heterochromic cyclitis: rubella virus antibodies and genome in aqueous humor. Am J Ophthalmol. 2004;138:46-54.

6. Guyard M, Perdriel G, Ducret J. [Apropos of a recent case of uveitis due to Q fever]. Bull Soc Ophtalmol Fr. 1959;7: 599-605, 599-605.

7. Kuhne F, Morlat P, Riss I, et al. [Is A29, B12 vasculitis caused by the $\mathrm{Q}$ fever agent? (Coxiella burnetii)]. J Fr Ophtalmol. 1992;15:315-321.

8. Million M, Halfon J, Le Lez ML, et al. Relapsing uveitis and optic neuritis due to chronic Q fever. Br J Ophthalmol. 2011; 95:1026-1029.

9. Rossiter-Thornton L, Rossiter-Thornton M, Azar D. Q fever-associated HLAB27 anterior uveitis. Clin Exp Ophthalmol. 2008;36:797-798.

10. Ruiz-Moreno JM. Choroidal neovascularization in the course of Q fever. Retina. 1997;17:553-555.

11. Drancourt M, Berger P, Terrada C, et al. High prevalence of fastidious bacteria in 1520 cases of uveitis of unknown etiology. Medicine (Baltimore). 2008;87:167-176.

12. Matonti F, Conrath J, Bodaghi B, et al. Uveitis in the course of Q-fever. Clin Microbiol Infect. 2009;15:176-177. doi: 10.1111/j.1469-0691.2008.02215.x. Epub;\%2009 Mar 11., 176-177.

13. Dijkstra F, van der Hoek W, Wijers N, et al. The 2007-2010 $Q$ fever epidemic in The Netherlands: characteristics of notified acute $\mathrm{Q}$ fever patients and the association with dairy goat farming. FEMS Immunol Med Microbiol. 2012;64: 3-12.

14. Niesters HG. Standardization and quality control in molecular diagnostics. Expert Rev Mol Diagn. 2001;1: 129-131.

15. Schneeberger PM, Hermans MH, van Hannen EJ, et al. Real-time PCR with serum samples is indispensable for early diagnosis of acute $\mathrm{Q}$ fever. Clin Vaccine Immunol. 2010;17:286-290.

16. Dupont HT, Thirion X, Raoult D. Q fever serology: cutoff determination for microimmunofluorescence. Clin Diagn Lab Immunol. 1994;1:189-196. 